\title{
'CELESTINA' DE FERNANDO DE ROJAS: DOCUMENTO BIBLIOGRÁFICO (decimonono suplemento)
}

\author{
Joseph. T. Snow \\ Randal Garza \\ Michigan State University
}

[Agradecimientos: Los siguientes amigos de esta revista o nos proporcionaron referencias a, o copias de, estudios anotados en este suplemento: E. Salas, H. L. Sharrer, H. C. Woodbridge, F. Maurizi, D. D. Severin, A. D. Deyermond, M. Rubio. E. Gascón Vera, y R. Soto.]

723. ALCALÁ FLECHA, Roberto. "La intención documental. Iconografía de la Celestina," en su Matrimonio y prostitución en el arte de Goya (Cáceres: Univ. de Extremadura, 1984), pp. 79-107 (8 ilustraciones).

Directa (en Rojas) o indirectamente (por ejemplo en "El arte de las putas" de N. Moratín), en Goya aflora el tema de la alcahueta y sus pupilas. Hay reflexiones de la celestinesca en los comentarios a los Caprichos 12, 15, 17, 31 y 36, y a unos ejemplos de los Álbumes.

724. ALLAIGRE, Claude. "Notule sur une autodéfinition de Calisto dans La Celestina ('yo, misto'). Les langues neo-latines, núm. 299 (1994): 17-22.

Pretende recuperar para el vocablo 'misto' su significado religioso de 'iniciado' (aquí en un culto melibeico): Calisto se siente indigno por ser corporal al lado de esta presencia angelical. 
725. ALONSO, Alvaro, "Huella de La Celestina en la ficción pastoril," en Medioevo y Literatura. Actas del $V$ Congreso de la Asociación Hispánica de Literatura Medieval, ed. J. Paredes (Granada: Universidad, 1995), 4 vols; vol. I: 251-260

Como modelo de lamento, como historia de amor y como fuente de conocimientos, varias obras del género pastoril encuentran en Celestina su claro eco: en La Diana (1563) de Alonso Pérez; en Ninfas y pastores del Henares (1587) de González de Bobadilla; en El prado de Valencia (1600) de Gaspar Mercader; y en Los pastores de Sierra Bermeja (1620) de Jacinto Espinel Adorno.

726. ATALA, Henri. "Se acabó el primer acto," en 'La Célestine: Comedia o Tragicomedia de Calisto y Melibea," (ver MAURIZI, 770), pp. 53-58

Rojas admiró el texto corto y completo del autor primitivo, el cual este autor sugiere pudiera titularse "El amante bobo y la alcahueta." Pero también reconoce que después compuso "tal fin que el principio desata," continuándolo y matando a sus protagonistas.

727. BACCHELLI, Franco. "Repertorio di opere antiche spagnole della biblioteca 'A. Panizzi' de Reggio Emilia." Quaderni di lingue $e$ letterature (Verona) 18 (1993): 59-103.

Los números 103 y 104 de su catálogo son ejemplares de la TCM (Milán: Zanotto da Castione, 1414; Venecia: F. di Allessandro Bindoni y Mapheo Pasini, 1531).

728. BELTRÁN, Rafael. "La huella de Tirant lo Blanc en la Celestina," Actas del III Congreso de la Asociación Hispánica de Literatura Medieval (Salamanca 1989), ed. M. I. Toro Pascua (Salamanca: Biblioteca Española del siglo XV - Dept ${ }^{\circ}$ de Literatura Española e Hispanoamericana, 1994). I:169-179.

Estudio comparado de Calisto y Tirant, de Plaerdemavida y Celestina (con retoños de Lucrecia) con motivo de mostrar como puede haber en por lo menos cuatro episodios del Tirant tantas semejanzas en la acción, la situación y la retórica para poder sugerir que eran estos episodios fuentes posibles para los autores de Celestina. 
729. BLANCO, Mercedes. "Las piezas liminares de la Célestine: un vestíbulo enigmático," en Actes du Colloque (ver MAURIZI, 770), pp. 119-143.

Rojas mismo nos ofrece pocos datos biográficos. En los paratextos celestinianos, sin embargo, algo se revela. El foco aquí es la carta "Del autor a un su amigo" que sirve de pórtico a Celestina, y cómo funciona en la obra como paratexto.

730. BOTTA, Patrizia. "Itinerarios urbanos en la Celestina de Fernando de Rojas." Celestinesca 18.2 (Otoño 1994): 113-131.

Estudio ilustrado y con buena bibliografía. Ofrece un panorama extenso (y visual!) de la ciudad y el espacio urbano donde se desarrolla la acción de la Tragicomedia (basado en numerosísimas referencias textuales). Ha creado Rojas la "idea" de la ciudad sin querer nunca especificar una particular.

731. " "Otra vez hacia una edición crítica de La Celestina (I)," en Actas del III Congreso de la Asociación Hispánica de Literatura Medieval, ed. M. I. Toro Pascua (Salamanca: Biblioteca Española del Siglo XV - Dept $^{\circ}$ Lit. Española e Hispanoamericana, 1994). II:953-963.

(Ver también, abajo, LOBERA, 776 y SCOLES, 790.) Habla de la necesidad primaria de una edición crítica de la Comedia de 16 actos $y$, por razones distintas, rechaza los stemmata propuestos independientemente por Rank (1978) y Marciales (1985). Propone nuevas posibilidades más lógicas según otras metodologías propuestas para llevar a cabo esta labor editorial.

732. . "Un esemplare annotato della Celestina e la traduzione inglese de Mabbe-I." Cultura Neolatina 52 (1992): 353-388. [Para la $2^{\mathrm{a}}$ parte, ver VACCARO, 797.]

Comentario al ejemplar plantiniana (1599) de Celestina y descubierta por la autora con centenares de glosas marginales en inglés (muy probablemente en la mano de James Mabbe). Presenta pormenorizados argumentos y análises que aclaran las relaciones entre los MSS de Alnwick (tempranas traducciones de Mabbe), el uso de la traducción italiana, el ejemplar glosado en inglés (ahora en la Biblioteca Nacional de Madrid), y la edición impresa de 1631 (la segunda versión de Mabbe). Con dos apéndices. 
733. BROWN, Kenneth. "A Seventeenth-Century Sephardic Reader's Negative Evaluation of Celestina." Celestinesca 18.2 (Otoño 1994): 151-154.

Es el Diálogo entre dos hermanos, texto sefardi supuestamente de Marruecos, y contiene una condena de Celestina y sus artes. El autor parece no saber que hubo una traducción hebrea de la obra en el siglo XVI por Samuel Tsarfati.

734. CARDENAS, Anthony J. "Cervantes's Rhyming Dictum on Celestina: Vita artis gratia or Ars vitae gratia?," Indiana Journal of Hispanic Literatures, no. 5 (Fall 1994): 19-36.

Estudio multifacético sobre los versos que Cervantes dedica a Celestina en el prólogo a su Quijote. Resultan ser una clave de su visión del papel que hace la imaginación en cualquier obra narrativa. Ofrece interesantes "lecturas" de la obra de Rojas también.

735. CARRASCO, Félix. "La recepción de Celestina a mediados del siglo XVI: evaluación de dos lecturas," en Actas del III Congreso de la Asociación Hispánica de Literatura Medieval (Salamanca 1989), ed. M. I. Toro Pascua (Salamanca: Biblioteca Española del siglo XV Dept $^{\circ}$ de Literatura Española e Hispanoamericana, 1994). 1:233240.

Lleva el concepto de la recepción de Celestina en la literatura posterior a 1) El Scholástico de Cristóbal de Villalón y 2) la Comedia de Sepúlveda, ambas obras del siglo XVI. Ilustrada con citas convincentes $y$, en el caso de Villalón, evidencia para una lectura contemporánea que prefiere la astucia de Celestina sobre la magia como explicación de la 'caída' de Melibea.

736. COMPANY COMPANY, Concepción. "Dos caminos encontrados para la posesión. Posesivo y artículo en el español antiguo." Romance Philology 68 (1994-1995): 111-135.

Estudia valores semánticos en dos construcciones posesivas en textos selectos entre 1490-1590 (tipo su casa de Juan vs. la casa de Juan), entre ellos Celestina. Llega meticulosamente a la conclusión que, en estos usos, la relación entre la comunicación y su forma no es arbitraria, sino que resulta ser bien motivada; la sintaxis sirve a la experiencia. 
737. . "De la gramática a la estilística: las duplicaciones posesivas en La Celestina," en Palabra e imagen en la Edad Media, eds. A. González, L. von der Walde, C. Company (México: UNAM, 1995): 141-156 (corrección de datos del número 583 del $17^{\circ}$ suplemento).

738. CORFIS, Ivy. A. "Judges and Laws of Justice in Celestina," en Studies on Medieval Spanish Literature in Honor of Charles F. Fraker, ed. M. Vaquero y A. Deyermond (Madison, WI: Hispanic Seminary of Medieval Studies, 1995): 75-89.

Presentación de varios pasajes sobre los juicios y castigos en Celestina: son explicaciones del texto rojano de parte del autor anónimo de Celestina comentada, MS anónimo de hacia mitades del siglo XVI. Las acciones explicadas aquí afectan la lectura de $\operatorname{los}$ actos $1,3,7,12,13$ y 14 .

739. CUETO, Sandra E. del. "The Role of the Fourth Deadly Sin in the Tragicomedia de Calisto y Melibea." Mester 22.1 (1993): 1-7.

Estudio de la envidia tal como se manifiesta en Pármeno y en Areúsa para determinar el papel de este pecado en la muerte de Calisto. Ofrece apoyo a la idea de que no se muere Calisto sólo como castigo de su 'loco amor,' sino que hay también agencias externas que impactan en su trágico fin.

740. DEYERMOND, Alan. "Keith Whinnom's Celestina Book," en Studies on Medieval Spanish Literature in Honor of Charles F. Fraker, ed. M. Vaquero y A. Deyermond (Madison, WI: Hispanic Seminary of Medieval Studies, 1995): 91-105.

Sobre el libro en el que Whinnom trabajaba durante el segundo lustro de los años 60 y que después abandonó, dejándonos una versión de la primera parte, unas ideas esbozadas de la segunda y sólo las intenciones con respecto a una tercera parte.

741. . "Hacia una lectura feminista de la Celestina," en Actes du Colloque (ver MAURIZI, 770), pp. 59-86.

Sugiere caminos de investigación para una lectura feminista de Celestina. Entre las muchas posibilidades sugeridas se destacan: el significado del título usado hoy; la representación tradicional de la mujer (la idealista y la misógina); la brujería, y el discurso femenino. 
742. FLORES CERVANTES, Marcela. "La autoría de La Celestina a la luz de un problema lingüístico: los pronombres objeto." Palabra $e$ imagen en la Edad Media, eds. A. González, L. von der Walde, C. Company (México: UNAM, 1995): 127-139 (corrección de datos del número 596 del $17^{\circ}$ suplemento).

743. FORCADAS, Alberto. "El Bursario (traducción de las Heroidas de Ovidio por Juan Rodríguez de la Cámara o del Padrón)," en Actas del X Congreso de la AlH, ed. A. Vilanova (Barcelona: PPU, 1992), vol. I: 179-188.

Postula que Rojas había conocido específicamente esta traducción porque tiene cosas no incluidas en Ovidio. Estos añadidos forman parte del texto de Celestina.

744. FOTHERGILL-PAYNE, L. "La cita subversiva en Celestina," en Actas del X Congreso de la $A I H$, ed. A. Vilanova (Barcelona: PPU, 1992), vol. I: 189-194.

Aligera el pesimismo de Celestina el arte de citar autoridades, a veces parodiándolas (Petrarca, Séneca, etc.). Confiere un subtexto irónico a - e intensifica la importancia de - la recepción de la obra de Rojas. Con ejemplos comentados.

745. GARCI-GOMEZ, Miguel. Calisto: sonador y altanero. Kassel: Reichenberger, 1994. $\left(^{*}\right)$

746. GARCIA, Michel. "Apostillas a 'Consideraciones sobre Celestina de Palacio."' Celestinesca 18.2 (Otoño 1994): 145-149.

Estudiando las 8 enmiendas principales del fragmento de Celestina, G. llega a considerar que el MS de Palacio es copia enmendada (por otro copista) de la versión primitiva, en la cual no intervino Fernando de Rojas.

747. GARCIA CÁRCEL, Ricardo, y J. BURGOS RINCÓN. "Los criterios inquisitoriales en la censura de libros en los siglos XVI y XVII." Historia social, número 14 (otoño 1992): 97-109.

Ilumina, en un estudio interno de los Indices de libros en el XVI y XVII, algunas curiosidades de los procedimientos de la Inquisición frente a los libros, entre ellas unas que tocan el caso de Celestina (ver 98, e ilustración, 105). 
748. GIMENO CASALDUERO, Joaquín. "La Celestina y su prólogo," en Actas del X Congreso de la AIH, ed. A. Vilanova (Barcelona: PPU, 1992), vol. I: 215-221.

Todos los personajes en la obra buscan el gozo de los premios de este mundo pese a que la arquitectura de la TCM (conflictos, disputas, oposiciones, contienda) sólo produce significados pesimistas. Dos momentos definitorios se encuentran en los actos 9 y 19 , aquí comentados.

749. GIRI, Donato. Il fondo antico ispanico della Biblioteca Civica di Verona, Col. Teatro del Siglo de Oro - Bibliografías y Catálogos 11, Kassel: Reichenberger, 1992.

Entre las entradas figura la traducción italiana de Celestina de Venecia: Gregorio de Gregorii, 1525 (p. 129).

750. GOMEZ MORENO, Angel, y Teresa JIMÉNEZ CALVENTE. "A vueltas con Celestina-bruja y el cordón de Melibea," en Actes du Colloque (ver MAURIZI, 770), pp. 201-228. Publicado también en Revista de filologia española 75 (1995): 85-104.

Estudio rico y sugerente que explora - en textos literarios y documentos de varia indole - las 'señas de identidad' hechiceriles, médicas, y de traficante en amores de Celestina. Abarca este estudio textos desde Plauto, pasando por "Trótula" y el Libro de las confesiones de Martín Pérez (h. 1415), y llegando a otras obras literarias y tratados científicos anteriores y posteriores a la obra de Rojas, aclarando e intensificando en cada apartado la complejidad de la figura de la alcahueta y sus multifacéticos oficios (todos ligados a la sexualidad). Merecen especial mención los comentarios al laboratorio celestinesco, el 'dolor de muelas' de Calisto y el cordón de Melibea.

751. GOSTAUTAS, Stasys. "Celestina a lo divino," en Homenaje a Justina Ruiz de Conde en su 80 cumpleafios, a cargo de E. Gascón-Vera y J. Renjilian-Burgy (Eria, PA: ALDEEU, 1992), pp. 97-111.

Ofrece un pormenorizado comentario a un personaje celestinesco de la Lima colonial, descrito en una obra satírica manuscrita llamada "La endiablada" por Juan Mogrovejo de la Cerda (h. 1626). 
752. GUTIÉRREZ, Jesús. "El celoso, entre La Celestina y el Decamerone," en Actas del X Congreso de la AIH, ed. A. Vilanova (Barcelona: PPU, 1992), vol. I: 233-240.

El celoso (olim La lena), de 1602 (con versión definitiva en Barcelona 1613), es una obra más cercana a Bocacio que la servil imitación de Celestina que algunos suponen.

753. HATHAWAY, Robert. "Fernando de Rojas' Pessimism: The Four Stages of Life for Women at the Margin." Celestinesca 18.2 (Otoño 1994): 53-73.

Marginadas desde el principio, las cuatro personajes - Lucrecia, Areúsa, Elicia y Celestina - forman una cadena de mujeres que se desintegra a lo largo de la obra. Se ve reflejada en cada una diferentes etapas de la vida de las mujeres al margen. Lo que comparten todas es el impulso erótico. Adicionalmente en el caso de la alcahueta, su muerte marca una vana lucha de la mujer marginada contra su destino.

754. HERRERA, Francisco José, "El rufián en el ciclo celestinesco: del sistema a sus variantes," en Medioevo y Literatura. Actas del V Congreso de la Asociación Hispánica de Literatura Medieval, ed. J. Paredes (Granada: Universidad, 1995; 4 vols), vol. II:477-487.

Toma el carácter rufianesco del colérico Centurio rojano y sigue la utilización de sus distintas características en la creación de Pandulfo (Segunda Celestina), Galterio (Comedia Thebaida) y Rampín (Lozana andaluza). La característica central, sin embargo, no varía: la sujeción de la mujer al rufián.

755. HEUGAS, Pierre. "La Celestina," en Jean Canavaggio, dir., Historia de la literatura española I. Edad Media (Barcelona: Ariel, 1994), pp. 238-260.

Discurre sobre los temas de siempre (género, tiempo, espacio, técnicas de acción, personajes, forma, estilo, influencia posterior) pero siempre ateniendo a la evidencia textual. Presenta ideas frescas y perspectivas interesantes en esta lectura tan amena como bien informada. Caveat lector: hay varias referencias erróneas a los actos. (¿hubo corrección de pruebas?). 
756. IGLESIAS-OVEJERO, Angel. "Ensayo de identificación de refranes y frases proverbiales en la Celestina," en Actes du Colloque (ver MAURIZI, 770), pp. 167-200.

Siguiendo las pistas de 5 estudios anteriores (O'Kane, Fernández Sevilla, Cejador, Le Rouge y Gella Iturriaga, y escarbando paremias en repertorios tempranos de refranes y sentencias (entre ellos figura el de Correas importantemente), aquí identifica y publica las 283 paremias ("unidad funcional de comunicación memorizada en competencia") de Celestina (utiliza la ed. Criado de Val/Trotter).

757. ILLADES, Gustavo. "La voz como diálogo o contienda en $L a$ Celestina," en Palabra e imagen en la Edad Media, eds. A. González, L. von der Walde, C. Company (México: UNAM, 1995): 327-337 (corrección de los datos del número 612 del $17^{\circ}$ suplemento).

758. IRUN DE SOJO, Gloria. "Catálogo gráfico descriptivo de la Imprenta del Molino de La Rovella." Tesis doctoral, Univ. de Valencia Dept $^{\circ}$ de Filología Española, 1994, 2 vols. Con ilustraciones.

En Vol. I (p. 259) y Vol. II.i (431-436) hay ilustraciones (textos y grabados, portada) de la ed. de Joan Navarro (Valencia 1575).

759. JAIME-RAMÍREZ, Hélios. "Le cômos dans la dramaturgie de la Célestine," en Actes du Colloque (ver MAURIZI, 770), pp. 111-118.

Explora los puntos de posible contacto entre obras de Aristófanes (como Las nubes y Las ranas) y Celestina para terminar especulando que posiblemente Rojas conociera, directa o indirectamente, estos textos del comediógrafo griego.

760. ___ "Comediografía en Aristófanes y en La Celestina." Mar Oceana. Revista del Humanismo Espanol e Iberoamericano (Zaragoza) 2 (1995): 223-228.

Presenta la continuación de una investigación suya emprendida en 1988. Aquí se trata de las características compartidas por la obra dramática de Aristófanes y Celestina. Aunque concede que el autor de Celestina no se haya inspirado directamente en Aristófanes, señala los rasgos constantes del comediógrafo griego que perduran hasta el siglo XV. 
761. JOSET, Jacques. "Notes sur La Célestine wallone de Marcel Hicter," en L'oeuvre en Wallon de Marcel Hicter (1918-1979). Mémoire Wallonne 2, Liége: Société de Langue et de Littérature Wallones, 1995, pp. 19-27 (ilustrado con 3 fotos de la producción de 1981).

Amplía los datos que el autor mismo proporcionó sobre el tema, publicados en Celestinesca 12.2 (1988): 67-72.

762. KELLY, Henry A. Ideas and Forms of Tragedy from Aristotle to the Middle Ages. Cambridge: Cambridge UP, 1993.

Libro sugerente que - en su apartado final (194-217) - comenta las acepciones de 'comedia' y tragicomedia' en varias obras del XV español $y$, entre ellas, Celestina. Rojas no las distingue claramente.

763. KISH, Kathleen V. "Celestina: estímulo multisecular," en Actas del X Congreso de la AIH, ed. A. Vilanova (Barcelona: PPU, 1992), vol. I: 249-254.

Valora el estudio de las posteriores Celestinas para iluminar la original $\mathrm{y}$, como ejemplos, estudia las traducciones italiana (Hordognez 1506), alemanas (Wirsung 1520, 1534), flamenca (anón. 1550), francesa (Lavardin 1578) e inglesas (Mabbe 1598, 1631) para dar un panorama de los ajustes. Semejantes 'libertades' no se encuentran ni entre traductores ni entre adaptadores modernos de la obra al escenario.

764. LEE, Charmaine. "La parodia perduta: un aspetto della letteratura castigliana medievale," en L'immagine riflessa. Testi, società, cultura = Dialettiche della parodia 1.1 (Torino: Ed. dell'Orso, 1992): 111-131.

Alega que no existe obra plenamente paródica en las letras medievales castellanas, pero le interesa parangonar y contrastar el contenido paródico del Libro de buen amor y Celestina (ambos con claras huellas didácticas). En Celestina, hay ricas parodias de géneros, de la comedia humanística, y de la 'religión de amor.'

765. LE ROUGE de RUSUNAN, Réjane. "La paremiología dans $L a$ Célestine." Tesis inédita de Troisième Cycle, Univ. de Nantes, 1985. $\left({ }^{*}\right)$

766. LOBERA SERRANO, Francisco, "Otra vez hacia una edición crítica de La Celestina (II)," en Actas del III Congreso de la Asociación 
Hispánica de Literatura Medieval, ed. M. I. Toro Pascua (Salamanca: Biblioteca Española del Siglo XV - Depto. Lit. Española e Hispanoamericana, 1994). II:965-974.

(Ver arriba, BOTTA, 731, y abajo, SCOLES, 790.) Rechaza el stemma que se propone Marciales (1985) para las ediciones de la Tragicomedia y presenta otro, construido a base de los errores conjuntivos y separativos, explicando sus novedades y su utilidad para una edición crítica de Celestina.

767. MAEZTU, Ramiro de. Don Quijote, Don Juan és Celestina: elfogult esszé fordíttota. Bucarest: Kriterion Könyvkiadó, 1988. 296 pp.

Traducción al húngaro de la obra ya clásica de Maeztu (1926) por György Jánosházy.

768. MARTYN, J. R. C., ed. António de Ferreira. The Comedy of Bristo, or The Pimp. Carleton Renaissance Plays in Translation 20, Ottawa: Dovehouse, 1990.

La obra traducida, Comédia do Fanchono ou de Bristo, se cita como obra influida por Celestina, hecho reconocido aquí aunque sin estudiar la cuestión.

769. MARTÍNEZ CRESPO, Alicia. "Salute e belleza femminili tra il Medioevo e il Rinascimento," en Nello spazio e nel tempo della letteratura. Studi in Onore di Cesco Vian (= La civiltà delle scritture 11, Univ. degli Studi di Parma - Fac. di lettere e filosofia) (Roma: Bulzoni, 1991), pp. 129-138.

Muchas de las costumbres en el uso de productos cosméticos vistas en Bocacio, Martínez de Toledo, Rojas, y Delicado están reflejadas en un MS de la Biblioteca Palatina de Parma, Manual de mugeres en el qual se contienen muchas y diversas reçeutas muy buenas (su texto es editado en Salamanca, 1995, por M. C.).

770. MAURIZI, Françoise, ed. La Célestine. Comedia o Tragicomedia de Calisto y Melibea. Actes du Colloque International du 29-30 Janvier 1993. Travaux et Documents, 2, Caen: Université-Maison de la Recherche en Sciences Humaines, [1995]. Rústica, 228 pp.

Con 10 estudios celestinescos, todos ellos reseñados en este suplemento. 
771. . "Le 'Tractado de Centurio'," en Actes du Colloque (ver MAURIZI, 770), pp. 87-109.

El punto de partida es que la interpolación es un avance del entremés a la vez que una subversión de lo trágico por los elementos de farsa, de parodia, de desajustes, etc., que la caracterizan. Los análises de Sosia y Centurio a la luz de lo cómico iluminan sus personajes y los hace más deleitables. Esta pequeña obra dramática no cumple con el propósito explícito de extender el idilio de los amantes. La trama de la venganza, tal como se realiza, estará lejos de la expectativa de los primeros lectores de la Tragicomedia.

772. MCGRADY, Donald. "The Problematic Beginning of Celestina." Celestinesca 18.2 (Otoño 1994): 31-51.

Hay varios asuntos entrelazados en el comienzo de Celestina que pretenden aclararse aquí: el MS que recibió, Rojas era trunco al comienzo y al final. Rojas decide aceptar el dialogo inicial - in media res - y presentar un primer encuentro entre dos jóvenes que se conocían. Era Rojas quien escribió (las razones aducidas aquí son más bien de estilo) el resumen del primer acto para orientar bien al lector (reteniendo el motivo del halcón). Y finalmente, Rojas crea un doble sistema temporal intencionadamente ambiguo que permite dos interpretaciones (Melibea enamorada al instante, o Melibea vencida por las ministraciones diabólicas de Celestina)

773. "'Entrando Calisto una huerta...' and Other Textual Problems." Hispanic Review 63 (1995): 433-440.

Aboga por un uso correcto transitivo de 'entrar' aquí (corregido en 'entrando en' por casi todos los editores desde la primera edición de la Tragicomedia) para dar el matiz de 'entrar por fuerza', matiz que tenía en textos posteriores. Cita ejemplos de este uso en latín, al lado de su empleo en castellano.

774. MIGUEL, Emilio de. "Autoría del acto 1 de La Celestina desde la perspectiva de la creación," en Actes du Colloque (ver MAURIZI, 770), pp. 19-51.

Reabre la cuestión de la autoría del primer auto. Su comparación del primer auto con los demás autos - escritos sin duda por Rojas - se basa en un análisis de la conducta de los personajes. 
Concluye afirmando que los "complejos personajes celestinescos" del primer acto se desarrollan con entereza propia a lo largo de la obra, lo cual favorece la autoría unitaria de Celestina.

775. MORENO HERNÁNDEZ, Carlos. "Diálogo, novela, y retórica en Celestina." Celestinesca 18.2 (Otoño 1994): 3-30.

Un excursus por el asunto del género de Celestina (concluye que es "antigenérico"). Aduce Platón (Banquete, República, lib. iii), Ortega, Bajtín, R. Gullón, F. Ayala y D. S. Severin (entre muchos otros) en una generosa apreciación de Celestina como eslabón (junto con el Quijote) en la cadena que produce la novela moderna. La discusión de Celestina es polifacética, enriquecedora.

776. MURILlO MURILlO, Ana. "Love and Chastity in Two Early English Versions of La Celestina," en Proceedings of the II National Conference of the Spanish Society for English Renaissance Studies (Oviedo: Universidad, 1992): 193-206. $\left(^{*}\right)$

777. NAYLOR, Eric. "La onomástica en Celestina," en Studia Hispanica Medievalia. IV Jornadas Internacionales de Literatura Espanola Medieval, Buenos Aires, 1993, ed. R. E. Penna y M. A. Rosarossa (Buenos Aires: Universidad Católica, 1995), pp. 120-127.

Como toma en cuenta los críticos anteriores, podemos llamar la discusión aquí - seria y sugerente - el último grito. Profundiza en las fuentes literarias de esos nombres conocidos. Termina negando rotundamente que el Libro de buen amor hubiera sido una influencia en Celestina (aparte de que no comparten nombres de personajes).

778. PRIETO DE LA IGLESIA, María Remedios, "Las piezas preliminares de La Celestina: un mensaje comunicacional," en Actas del III Congreso de la Asociación Hispánica de Literatura Medieval, ed. M. I. Toro Pascua (Salamanca: Biblioteca Española del Siglo XV Depto. Lit. Española e Hispanoamericana, 1994). II:797-803.

Según este estudio, las estrofas 4-7 de los versos acrósticos eran las únicas originales (son el "bajo fin" referido en la "Carta") y no identifican ni el autor ni su procedencia; al contrario son tan anónimas como. los "papeles" del autor primitivo. Es la elaboración posterior del borrador por Rojas que resulta en la expansión de los versos acrósticos, tan pertinentes a su 
publicación en la Comedia. Este estudio se continúa en otro de Sánchez Sánchez-Serrano (ver 789, abajo).

779. RAMOS, Juan Antonio. Papo Impala está quitao. Río Piedras, PR: Ed. Huracán, 1983. 91 pp.

Un monólogo dramático que incorpora una narración - en un puertorriqueño coloquial - de los sucesos ocurridos en la Celestina de Rojas (39-48). Una perspectiva original, cómico.

780. RODRÍGUEZ, Juan Carlos. "El caso de La Celestina," en su La literatura del pobre (Granada: de guante blanco/Comares, 1994), pp. 65-109.

Celestina plantea textualmente la matriz animista que señala las nuevas relaciones personales. Es un animismo humanista que celebra el amor como amistad entre iguales y refleja la nueva burguesía mercantil. El amor específicamente retratado es la loca pasión (desacralizada, desde luego) que tiene como medio la "ganancia" vista desde la perspectiva de una ideología que identifica a los individuos como libres, pero sólo asociándose libremente en clases (los privilegiados con otros privilegiados, los pobres con los pobres). Este animismo humanista se distancia del orden feudal y sacral.

781. RODRIGUEZ-PUERTOLAS, Julio. "Amor, sexualidad y libertad: la mujer en la literatura castellana del siglo XV," en Discurso erótico $y$ discurso transgresor en la cultura peninsular. Siglos XI al XX, coord. M. Díaz-Diocaretz y I. M. Zavala, Col. Investigación y Crítica 8, Madrid: Tuero, 1992, pp. 29-55.

Analiza Celestina a la luz de su presentación de mujeres atípicas dentro de las letras del siglo XV. En contraste con la represión ejercida por una sociedad machista, la mujer moderna (estudia Areúsa y Melibea) manifiesta una nueva libertad por medio de la franca expresión de sus emociones.

782. ROJAS, Fernando de. A Celestina. Tradução e notas de José Bento. Colecção Amadis 4, Lisboa: Assírio \& Alvim, 1988. xvii + 201 pp.

Excelente introducción densa, una breve bibliografía, el texto completo de la TCM (la base es la ed. de Severin en Alianza) y, al final, 64 notas. Con ilustraciones de ediciones antiguas. 
a. Colóquio-Letras, no. 115 (1990): 190-92, S. Reckert.

783. - LC. Col. Fontana, Clásicos universales, 1, Barcelona: Edicomunicación, 1994. Rústica, 282 pp. Capa ilustrada.

Para estudiantes. contiene la TCM completa en ed. de Jesús Ibánez Campos, un estudio preliminar (7-31) por Francesc-Lluis Cardona Castro, y una bibliografía de ediciones (32-35). Las notas son mínimas (265-280, del mismo editor).

784. - LC. Grandes Autores-Literatura Universal, Barcelona: Edicomunicaciones, 1994. Enc., 221 pp.

Idéntico contenido (ver entrada anterior) en otro formato.

785. Selestina, en Plotovskoï roman. Introd. de N. Tomashevskogo. Traducción de E. Lyssenko y S. Piskunovy (Moscú: Pravda, 1989), pp. 21-202.

Traducción rusa de la Celestina (TCM).

786. ROMERA CASTILLO, José. "La Celestina de Rojas/La Celestina de Torrente Ballester," en Homenaje a H. López Morales, ed. M. Vaquero y A. Morales (Madrid: Arco Libros, 1992): 265-284.

Un cotejo útil de la obra de Rojas y la adaptación de T.B. (estrenada en 1988 por la Compañía Nacional de Teatro Clásico). Analiza los procesos creativos utilizados en la producción de esta "bella versión celestinesca."

787. RUSSELL, Peter E. "El problema de lo inconsecuente textual en $L a$ Celestina," en Actes du Colloque (ver MAURIZI, 770), pp. 9-18.

En Celestina hay enigmas, ambigüedades $\mathrm{e}$ incertidumbres. ¿Cómo explicar estas incongruencias? $R$. concluye que ni la Comedia ni la Tragicomedia tenian pretensiones de ser completamente claras o racionales. Más bien, el texto se debe considerar experimental en cuanto a ciertos procedimientos estilísticos.

788. SALUS, Carol. "Picasso's Celestina Knitting." Celestinesca 18.2 (Otoño 1994): 133-144. 
El análisis del dibujo de Picasso sugiere que la presencia del gato señala una alusión a su oficio de hechicera, lo cual añade un tercer oficio a los dos reconocidos en este dibujo por otros: el de labrandera y el de alcahueta.

789. SÁNCHEZ SÁNCHEZ-SERRANO, Antonio, " Diálogos interpolados o refundidos en la Comedia de Calisto y Melibea," en Actas del III Congreso de la Asociación Hispánica de Literatura Medieval, ed. M. I. Toro Pascua (Salamanca: Biblioteca Española del Siglo XV Dept $^{\circ}$ Lit. Española e Hispanoamericana, 1994). II:805-812.

Extensión de otro estudio (ver 778, arriba). Aquí se examinan diálogos de la Comedia que muestran haber sido "entretallados" a base de cortes y soldaduras que delatan el proceso de una elaboración en que a veces parece dudosa la lógica.

790. SCOLES, Emma, "Otra vez hacia una edición crítica de La Celestina (III)," en Actas del III Congreso de la Asociación Hispánica de Literatura Medieval, ed. M. I. Toro Pascua (Salamanca: Biblioteca Española del Siglo XV - Dept $^{\circ}$ Lit. Española e Hispanoamericana, 1994). II:947-952.

(Ver también, arriba, BOTTA, 731, y LOBERA, 766). Presentación de ciertos problemas metodológicos e interpretativos de la edición de Marciales. Anuncia la preparación de una edición con criterios más amplios que los de Marciales.

791. SCHMIDHUBER, Guillermo. "Indagación autoral en La segunda Celestina, una comedia de Sor Juana Inés de la Cruz y de Agustín de Salazar y Torres." Cahier d'Etudes Romanes 17 (1993): 179-191.

Afirmación de Sor Juana como coautora de La segunda Celestina (siglo XVII). Las pruebas para ello residen en sus análises textuales de uno y otro co-autor.

792. SEVERIN, Dorothy S. Witchcraft in 'Celestina'. Papers of the Medieval Hispanic Research Seminar, 1, Queen Mary-Westfield Coll., Dept. of Hispanic Studies (London), 1995. 58pp. Portada ilustrada.

En torno a Celestina hay toda una serie de referencias que le atribuyen la práctica de brujería. Se investigan sistemáticamente en este estudio las relaciones Celestina-diablo. El último éxito de ésta como tercera se vincula a los conocimientos de la magia negra. Celestina funciona en un mundo esencialmente masculino 
con cierta autonomía aunque se la presenta con potencia invertida. Su muerte, después de un efímero éxito como mujer, señala el fracaso de este universo-al-revés que ella simboliza.

a. Celestinesca 19 (1995): 93-104, M. da Costa Fontes.

793. SNOW, Joseph T. "Fernando de Rojas as First Reader: ReaderResponse Criticism and Celestina. Studies on Medieval Spanish Literature in Honor of Charles F. Fraker, ed. M. Vaquero y A. Deyermond (Madison, WI: Hispanic Seminary of Medieval Studies, 1995): 245-258.

Basándose en las ideas de Wolfgang Iser, S. propone que Rojas, al incorporar en los preliminares la 'historia' de sus propias lecturas de los 'papeles' del autor primitivo, crea el modelo paradigmático para las futuras lecturas de la Tragicomedia. Además, su propia ficcionalización se ve como una manera de proteger lo que es una lectura subversiva de la sociedad que Rojas tan bien conocía.

794. $\longrightarrow$ y Randal GARZA. "Celestina de Fernando de Rojas: documento bibliográfico (decimoctavo suplemento)." Celestinesca 18.2 (Otoño 1994): 163-176.

Ampliación en 53 entradas de la bibliografía de Snow (1985).

795. SOTO RIVERA, Rubén. "El secreto de Celestina," en su Consideraciones tempestivas acerca de 'La Celestina' y 'La hora de todos' y 'La fortuna con seso' (Puerto Rico: Autor, 1995), pp. 4-45.

Un estudio que aboga por una lectura de Fortuna en Celestina con una dimensión más temporal que espacial (no acepta la propuesta de S. Gilman). Las ramificaciones son varias y la metodología se centra en una serie de asociaciones, a veces etimológicamente propulsadas y otras veces poéticamente intuidas, no siempre claras o bien organizadas. Celestina es abeja; es discreta, mensajera del amor erótico, aprovechadora de oportunidades, guardadora de secretos, causadora intencionada de Eros y a la vez, casualmente, de Thanatos, pero siempre respetadora del 'secreto': que el usar bien de ocasiones es manipular expertamente el Buen Tiempo. $Y$ en eso radica su superioridad. 
796. . "Celestina caira," en su Consideraciones tempestivas acerca de 'La Celestina' y 'La hora de todos' y 'La fortuna con seso' (Puerto Rico: Autor, 1995), pp. 46-92.

La palabra germanesca 'caire, caira' no aparece en Celestina. Como significaba, entre otras cosas, 'pago de la prostitución,' se vincula ideológicamente con la obra. Aquí se exploran éste y otros matices de palabras relacionadas ('cara,' 'cairel,' 'cairelar') al pretender establecer una base casi etimológico-filosófica para las iniciativas y las consecuencias de las acciones de la alcahuetalapidaria-prostituta-costurera (y más).

797. VACCARO, Elisabetta. "Un esemplare annotato della Celestina e la traduzione inglese di Mabbe-II." Cultura Neolatina 52 (1992): 389-419.

Después de la presentación panorámica de $\mathrm{P}$. Botta (ver arriba, 732), en esta segunda parte vemos un muestrario de ejemplos de las coincidencias, divergencias, traducciones malas, omisiones, etc. que se tienen que estudiar para vincular las glosas marginales en inglés del ejemplar de la BN (Madrid) de la ed. plantiniana de 1599 a la versión impresa de la traducción inglesa de Mabbe (1631).

798. VERMEYLEN, Alfonso. "La Celestina, objeto de una encarnada sospecha de judaísmo," en Studia Hispanica Medievalia. IV Jornadas de Literatura Espanola Medieval, Buenos Aires, 1993, ed. R. E. Penna y M. A. Rosarossa (Buenos Aires: Universidad Católica, 1995), pp. 215-222.

Pretende rebatir la insistencia con que se ha mantenido la identificación del 'Bernardo' (Celestina, auto I) con el de Claraboya (Forcadas 1973, 1978) y la identificación del autor del primer auto con una actitud judaizante que es la de Rojas. Apoya la identificación del 'Bernardo" con el del Corbacho (Bernardo de Cabrera) e insiste el autor en su propia identificación del autor del primer auto con un clérigo toledano anónimo (argumento propuesto en 1983) que no pudo ser Rojas.

799. VIAN HERRERO, Ana. "El Diálogo intitulado El Capón tras la huella de Celestina: una vez más, una cuestión de género." Celestinesca 18.2 (Otoño 1994): 75-111. 
Extenso y pormenorizado estudio de este diálogo híbrido (hay analogías con Celestina) en donde el diálogo y la narración se subordinan a una acción dramática. Considera también el marco menipeo, los modelos terencianos y celestinescos, el papel de la parodia y mucho más.

800. WALTHAUD, Rina. "La fortuna de Dido en la literatura española medieval (desde las crónicas alfonsíes a la tragedia renacentista de Juan Cirne)," en Actas del III Congreso de la Asociación Hispánica de Literatura Medieval (Salamarica, 3-6 octubre de 1989), ed. M. I. Toro Pascua (Salamanca: Biblioteca española del siglo XV Dept $^{\circ}$ de Literatura Española e Hispanoamericana, 1994), pp. 1171-1181.

En las pp. 1177-1179 repasa la figura de Dido en la literatura medieval y cómo llega a figurar en la Tragicomedia, y cómo ésta, en su turno, deja su huella en la influencia de la Tragedia de los amores de Eneas y de la Reyna Dido de Juan Cirne (mediados del s. XVI).

801. WHINNOM. Keith. Medieval and Renaissance Spanish Literature. Selected Essays, ed. A. Deyermond, W. Hunter, y J. T. Snow (Exeter: Exeter Univ. Press \& Journal of Hispanic Philology, 1994. xli +228 pp.

Republicación de dos estudios celestinescos de Whinnom (1980, 1981).

a. Celestinesca 18.2 (Otoño 1994): 155-161, J. C. Conde López;

b. La corónica 23.2 (Spring 1995): 141-143, I. A. Corfis;

802. ZIMMERMAN, Marie-Claire. "Le dire dans la Célestine: pouvoir, plaisir et solitude," en Actes du Colloque (ver arriba, MAURIZI, 770), pp. 145-166.

La atracción, el poder, y la amenaza del lenguaje (oral) hace de Celestina un texto muy consciente de su misma fragilidad. Aquí, a la luz de varias facetas del lenguaje vivo (ejemplos agudísimos sacados de los actos 17 [seducción de Sosia], 10 [el poder del nombre 'Calisto'], 7, 11, 19), se exploran algunas de sus posibilidades. 